\title{
Feasible Series Compensation Applications using Magnetic Energy Recovery Switch (MERS)
}

\author{
Jan A. Wiik, Takanori Isobe, *Taku Takaku, F. Danang Wijaya, Kazuhiro Usuki, \\ Nobuyuki Arai and Ryuichi Shimada \\ Tokyo Institute of Technology \\ N1-33, 2-12-1 O-okayama, Meguro-ku, Tokyo, Japan \\ Email: jan.wiik@nr.titech.ac.jp \\ *Fuji Electric Device Technology Co., Ltd. \\ 4-18-1, Tsukama, Matsumoto, Japan
}

\section{Keywords}

$<<$ Converter circuit $>>,<<$ Electronic ballast $>>,<<$ FACTS $>>,<<$ Generator excitation system $>>$, $<<$ Power factor correction $>>$

\begin{abstract}
Different series compensation applications by using the Magnetic Energy Recovery Switch are reviewed. The Magnetic Energy Recovery Switch is a variable series compensation device characterized by simple configuration and control as well as a large operating range. Three different applications areas are introduced; Load control, generator power capability improvements and series compensation in transmission systems. Load control application seems promising for cases where control of the frequency is not a necessity, such as for fluorescent lamps. By using MERS in series with permanent magnet generators can the output power capability of the generator be increased. At the high power end, MERS is discussed for use as a series compensator in transmission systems, where the power flow can be controlled and increased. In summary, the paper suggests a wider use of series compensation in electrical systems.
\end{abstract}

\section{Introduction}

Use of series compensation in electrical systems has a long history with applications in the low voltage to the high voltage range. In weak distribution grids, series compensation is used as an effective way to improve the voltage profile during variable loading conditions and also to reduce voltage flicker resulting from e.g. starting of induction motors. For the transmission systems, the series capacitor has been used to increase the transmission capacity of long power lines by reducing the equivalent reactance.

The Magnetic Energy Recovery Switch (MERS) has been suggested as a new variable series compensator. With a simple configuration using line frequency switching, low losses and low cost implementation is possible. The purpose of this paper is to review and investigate applications of this new series compensation configuration. The first part of the paper introduces the MERS and its operation. The second part describes some possible MERS applications including experimental results. This is followed by a discussion and a conclusion.

\section{Magnetic Energy Recovery Switch (MERS)}

The Magnetic Energy Recovery Switch (MERS) consists of 4 forced commutated switches and a small dc-capacitor [1]. The configuration is similar to that of a single phase full bridge, but the control is different and the size of the capacitor is several times smaller. By controlling the path of the current through the device (Fig. 1), the size of the injected series voltage can be controlled. Examples of resulting curves are shown in Fig. 2. Three modes of operation are indicated. The not-continuous and the dc-offset mode results by turning two and two switches on/off in pairs while the active by-pass mode results when actively by-passing the capacitor for a part of the period by using pattern Fig. 1c. The 

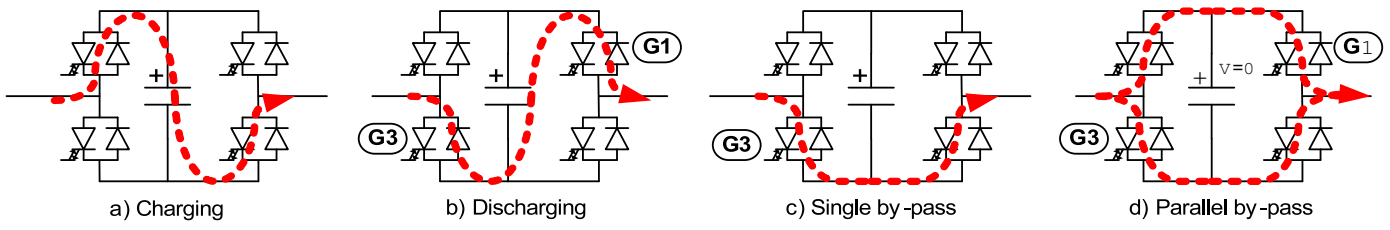

Figure 1: Possible current paths through the MERS when current is going from left to right.

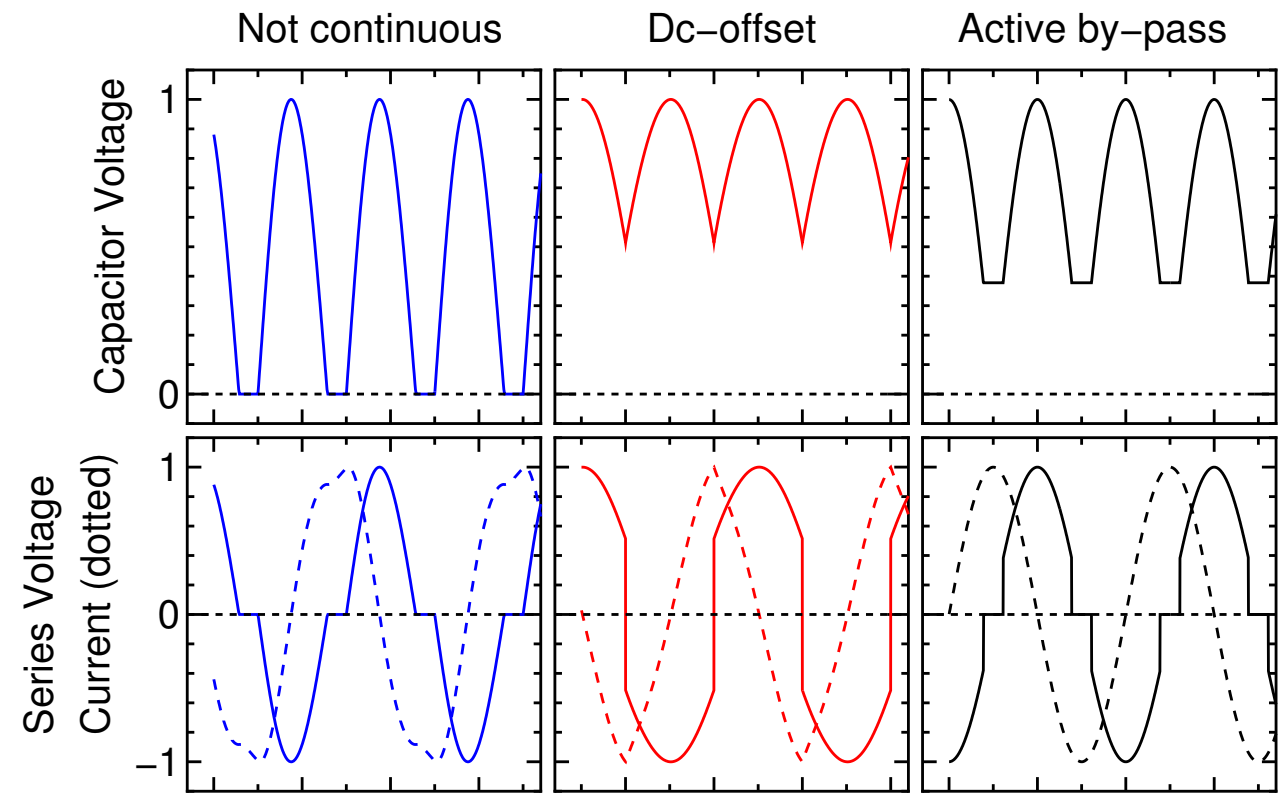

Time

Figure 2: Example of resulting voltage and current curves when controlling the MERS.

resulting current wave form depends on the application; however, it will always be 90 degrees to the injected series voltage. Important features of the MERS operation are:

- Line frequency switching, one switch is only turned on and off once during an electrical cycle.

- The size of the capacitive injected series voltage can be varied from zero to rated voltage within the current rating of the device.

- The series voltage injection capability stays constant even with varying frequency.

- Losses are similar to if the current continuously goes through one diode and one active switch.

- Harmonic distortion changes dependent on control set-point.

- When operated in dc-offset mode, the control of gate signals will automatically control the phase of the current [2].

Depending on the application, the control will change. Details regarding this are included in the application section.

\section{Series compensation applications using MERS}

\section{Load control}

With the coming of power electronics and power converters, new load control applications were developed, such as motor drives. The purpose of these power converters is to control the voltage magnitude and also the applied load frequency. However, in cases where only load voltage control is needed, there might be potential for using simpler series compensation devices. 

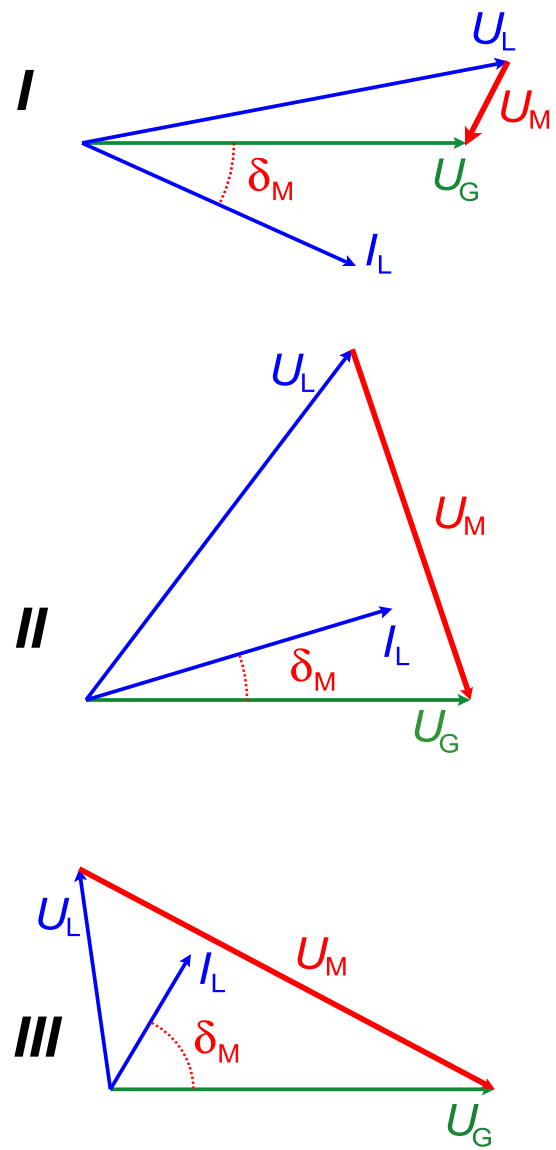
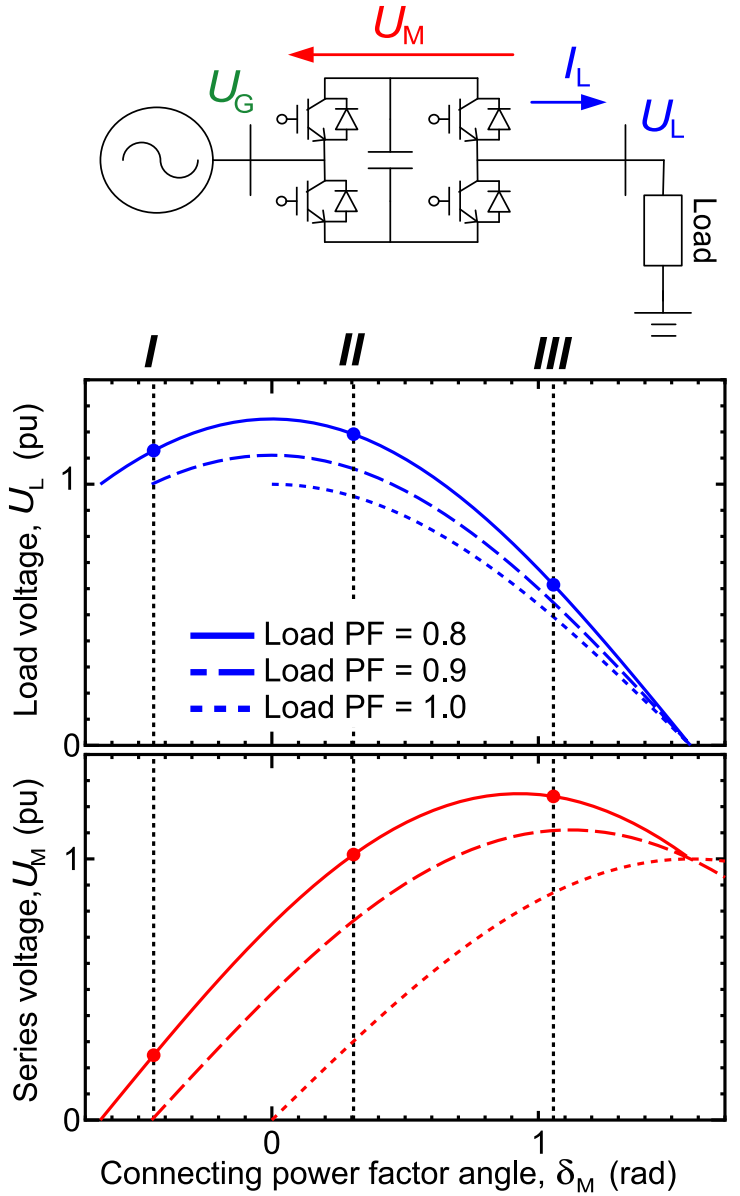

Figure 3: Illustration of load voltage control with MERS. The load voltage can be controlled by controlling the phase of the current in relation to the connecting grid voltage.

Load control using series compensation is illustrated in Fig. 3. The load voltage can be controlled from a maximum value to zero. The power factor is reduced for load voltages lower than rated and will be in the leading area. The load voltage and connecting power factor can not be controlled independently.

The control of the MERS according to the illustrated principle is very simple. A possible implementation is to identify the zero crossing of the grid side voltage, and vary the gate signals accordingly. Two and two switches are switched on and off in pairs. By doing this, the direction of the injected voltage phasor can be controlled [3]. Light intensity control of fluorescent lamps is used in the following to illustrate the operation of the MERS circuit for load voltage.

Light intensity control of fluorescent lamps is difficult with the use of triacs due to zero current periods. Several electronic ballast configurations exists, however with increased complexity. These solutions are normally based on high frequency switching and the use of reduced sized magnetic ballast, meaning that existing lamp installations not can be used directly. For reduced power output of fluorescent lamps(dimming) it has been suggested that the efficiency with normal magnetic ballasts is higher than that for electronic ballasts [4]. This indicates the feasibility of lamp dimming by controlling the applied voltage and not the frequency.

MERS is investigated as such a possible solution by using the configuration in Fig. 4. The experimental results showed that the light intensity could be controlled by using the MERS. Stable light output could be achieved down to an input power of 50 percent. Experimental results are shown in Fig. 5. Some observations are:

- Some distortion in the current wave form.

- Power factor is improved for increasing input power.

- The relative losses are reduced for reducing power outputs. Control power of approximately $1 \mathrm{~W}$ is not included in the graph.

- The light intensity can be controlled by simple phase-shifting the gate signals according to the input voltage. 


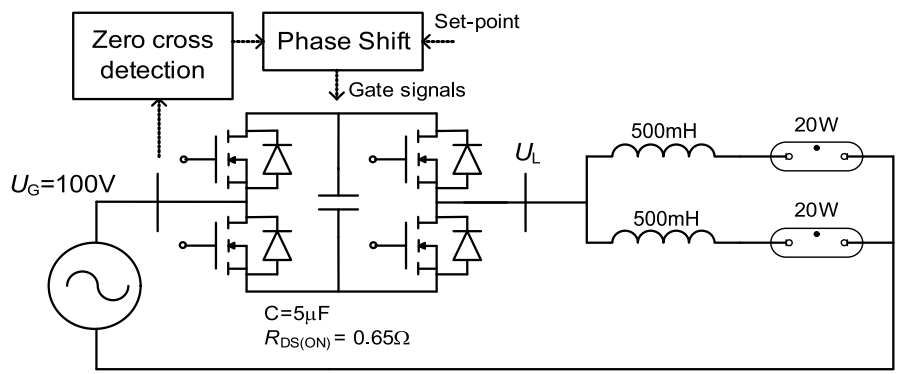

Figure 4: Configuration of experimental set-up used to study the use of MERS for controlling light intensity of fluorescent lamps.
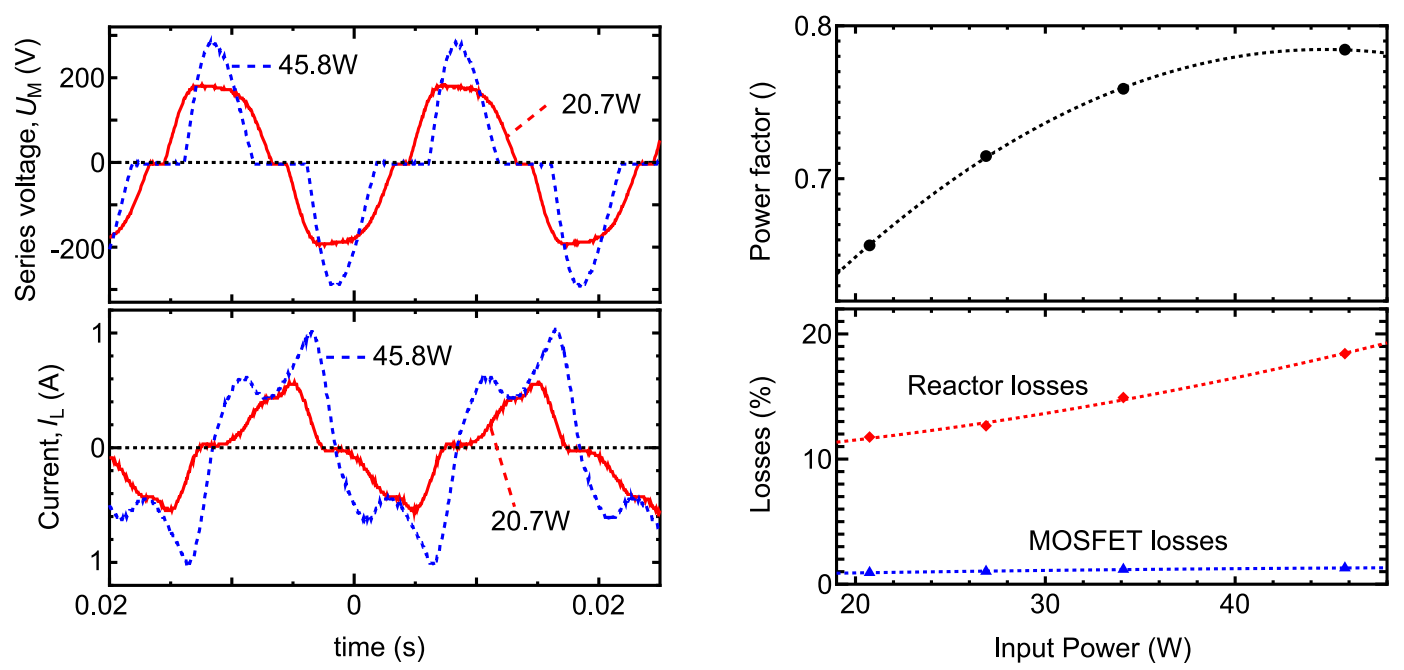

Figure 5: Resulting curves when using MERS to control the light intensity of the lamp. Left side shows resulting curves for two different power outputs. The right side shows variations in power factor and losses as a function of input power.

Due to the use of line frequency switching and MOSFETs, the gate drive can be simplified and cost reduced. Photovoltaic output devices (TLP190) were used for the experiments, having relatively slow but sufficient response for this application.

During start-up of fluorescent lamps, large currents may occur. To protect the MERS device from high capacitor voltage, the MERS device is by-passed during this period by using current path given in Fig. 1c or Fig. 1d.

The experiments were performed with glow starter type lamps. By using rapid starter type lamps with initial unity power factor, the resulting power factor and current distortion will also improve. This is due to the reduced size of the series injected voltage for a given load voltage. The distortion can also be improved by the selection of capacitor size. A detailed discussion regarding this is beyond the scope of this paper.

\section{Generator output power improvements}

Synchronous generators are characterized by a large synchronous reactance. Due to the resulting voltage drop across the reactance, the excitation level must be increased when the output power increases. The MERS has been suggested as a way to increase the maximum output power level for a given field excitation [5][2]. This can be performed by compensating the whole or parts of the voltage drop across the synchronous reactance.

Fig. 6 illustrates the principle of MERS control in combination with synchronous generator (generator parameters used are the same as for experimental set-up). The left part shows the resulting phasor diagrams for the case without MERS and with two different MERS controls. For the case without the MERS, it is clear that there is a substantial voltage drop due to the synchronous reactance, especially for increasing currents. One suggested use of MERS is to control the generator voltage to 1 per unit (constant voltage control). This means that only parts of the voltage drop across the synchronous reactance is cancelled. Another method is to control the current such that it is oriented according to the internal 


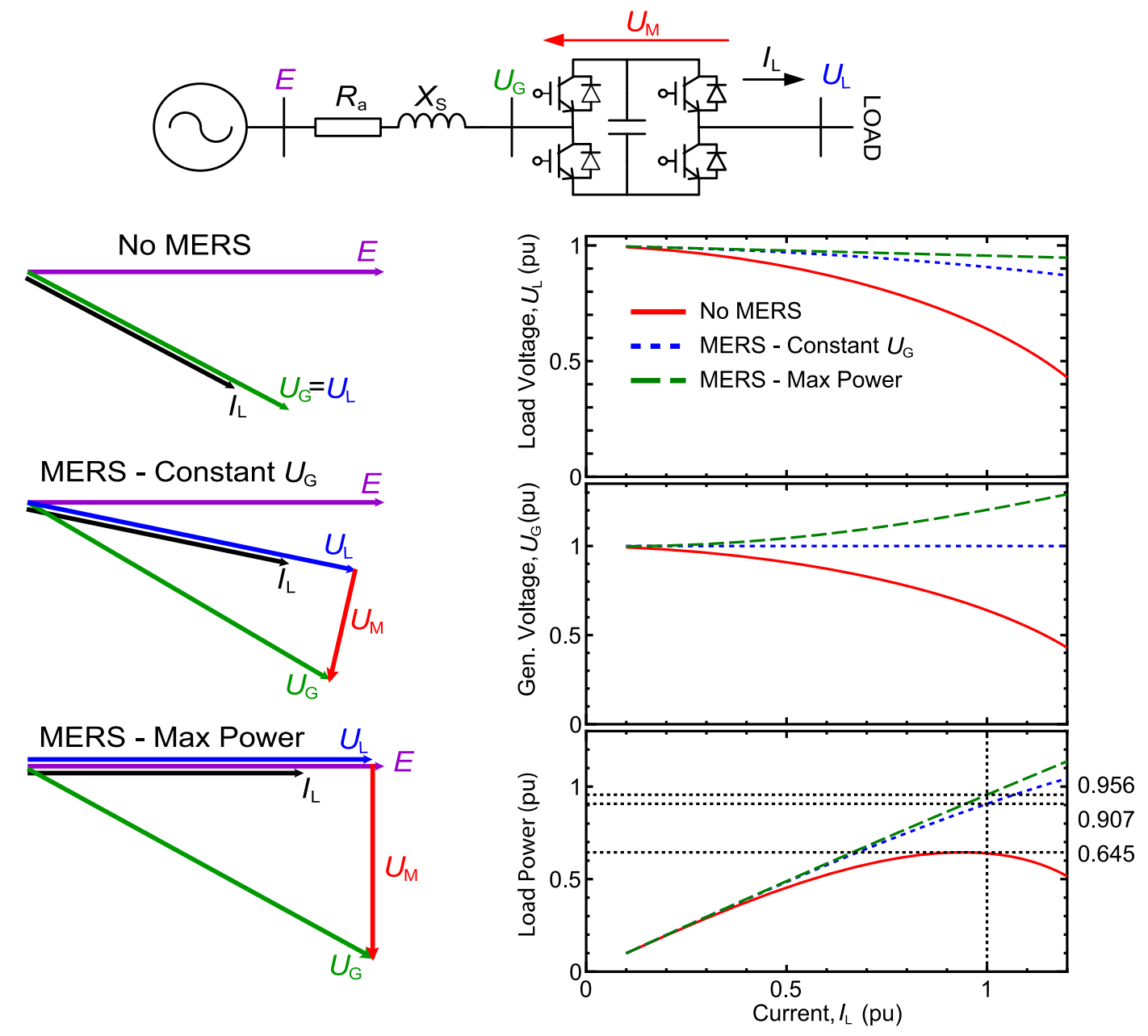

Figure 6: Illustration of principle when using MERS to improve the output power capability of synchronous generators.

generator voltage, $E$. This results in maximum power capability for a given output current(maximum power control). However, the result is a higher terminal voltage with possible need for special design.

The right part of Fig. 6 compares the three cases for different currents and keeping constant no load excitation. When analyzing the steady state power capability, the current is not allowed to exceed the rated current. With this constraint, the maximum power capability is increased with around 40 percent for the case with MERS and constant voltage control, and with around 48 percent for the case with maximum power control. The maximum power output for the case without MERS occurs before rated current is reached. On the other hand, by increasing the current above rated current and by using MERS control, the output power can be further increased. Based on this, the MERS can be said to be able to increase the short term over-load capacity of the generator substantially and well above the steady state power capacity.

Some experiments were conducted in order to verify the output power improvements by using MERS. The set-up is shown in Fig. 7 , and constant voltage control of the MERS was used. The excitation was adjusted during no-load such that rated voltage was achieved. After this, constant excitation current was kept. The purpose using constant excitation was to imitate the characteristics of a permanent magnet generator. As a result, the voltage at rated current with no MERS is reduced as well as the power rating of the generator.

The experimental results are shown in Fig. 8. Due to the use of a diode bridge as an active load, there is substantial distortion in the waveforms. The right part of the figure summarizes the results for the case with and without MERS. The case with MERS shows output power improvements of around 34 percent compared to the case without MERS.

The estimated values shown in Fig. 8 are based on ideal conditions with pure sinusoidal waveforms and only considering the synchronous reactance. In the real case, the waveforms are distorted due to the diode bridge. Additionally, a dc-current excitation type synchronous generator was used. Due to the field 


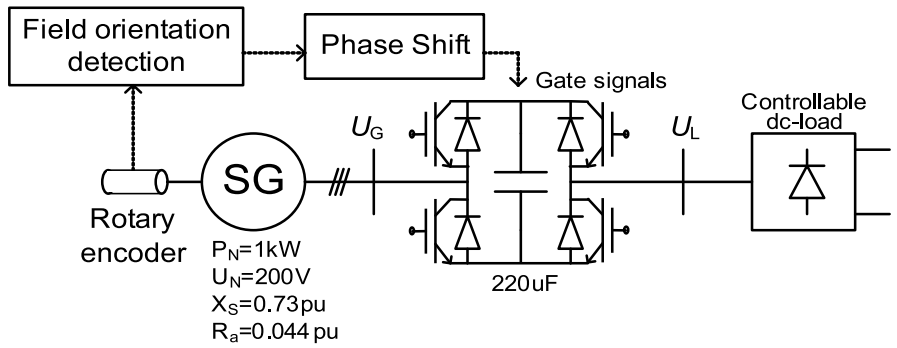

Figure 7: Configuration of experiment performed with synchronous generator and MERS.
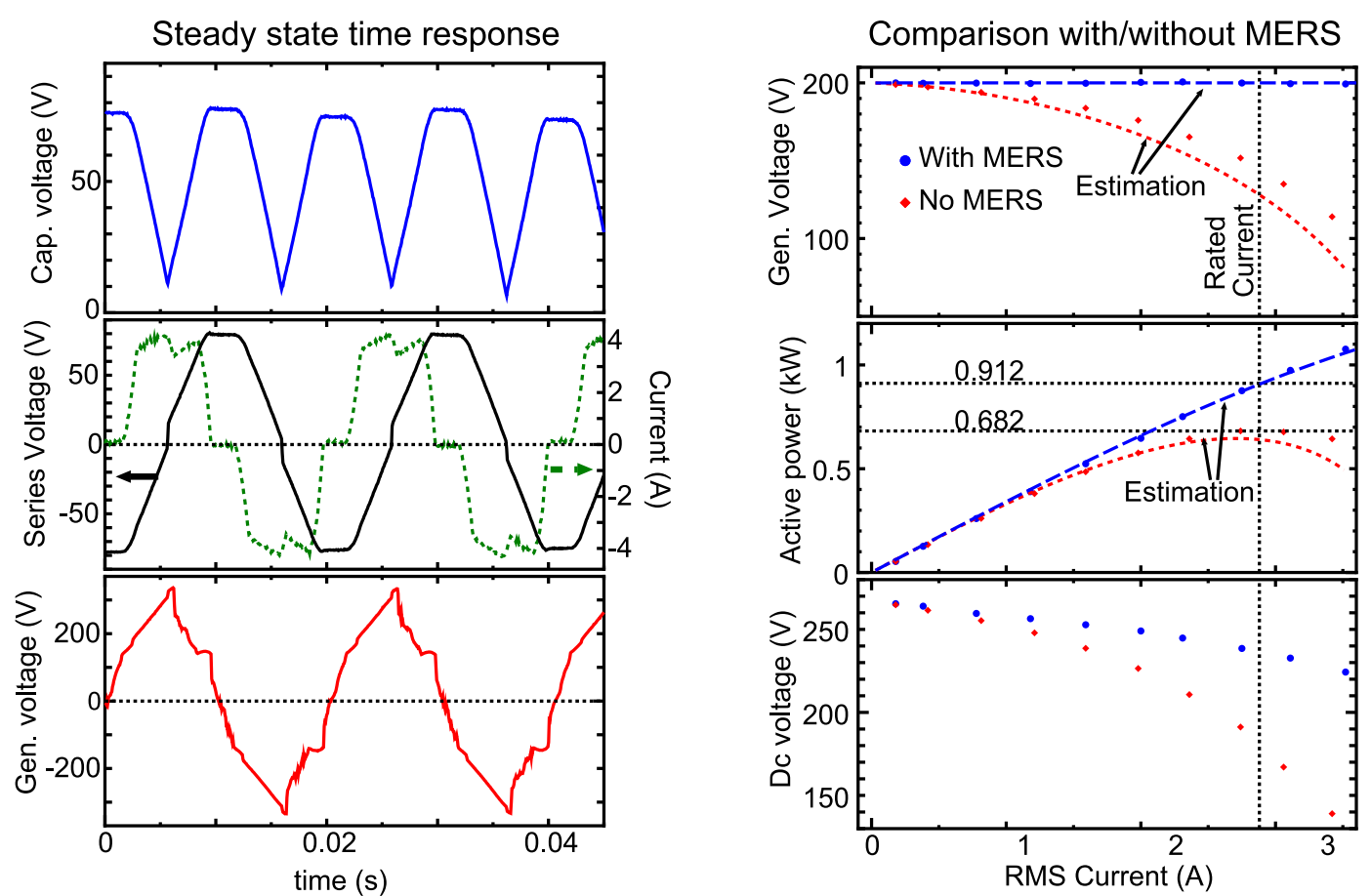

Figure 8: Experimental results with synchronous generator, MERS and diode load. Left side shows time trends, while the right side shows results when applying different sized dc-currents at the dc-load.

windings, the transient reactance will be smaller than the synchronous reactance. These two factors are believed to be the reason for the deviation between the estimated and actual experimental data.

Characteristics when using the MERS for power capability improvements of synchronous generators can be summarized as:

- The steady state power capability is increased by canceling parts or all of the voltage drop across the synchronous reactance in cases with constant field excitation (e.g. permanent magnet generators)

- The short term power capability can be increased substantially.

- Difference in power capability improvement resulting from MERS control with constant generator voltage control or maximum power control is not large.

- Maximum power control of MERS results in increased generator terminal voltage, meaning constant generator voltage control might be an attractive solution.

- The capability of the MERS to operate during variable frequency makes the configuration especially suited for applications such as wind power generators.

\section{Series compensation in transmission systems}

Series compensation is typically used to improve the transmission capacity and to control the flow of power in the case of parallel transmission paths. Series compensation have a long history in transmission 


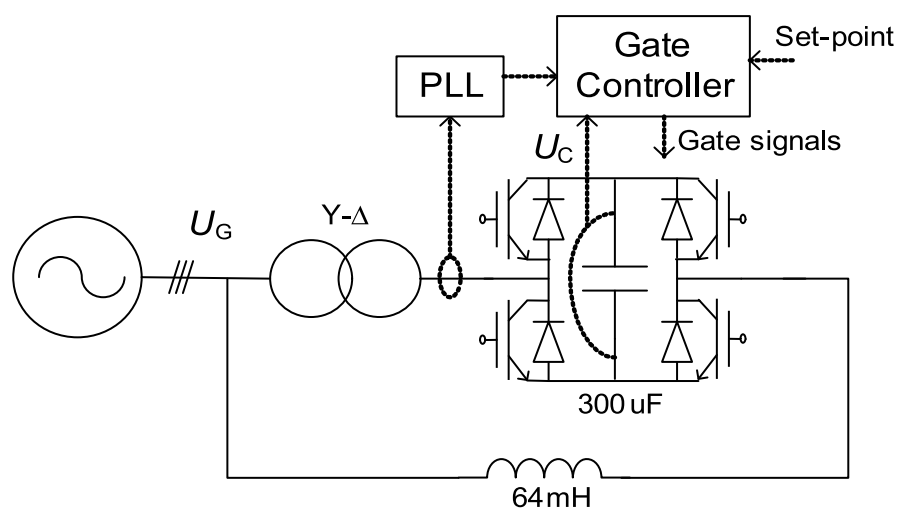

Figure 9: Configuration of experimental set-up used to study the use of MERS for controlling the power flow in transmission systems.
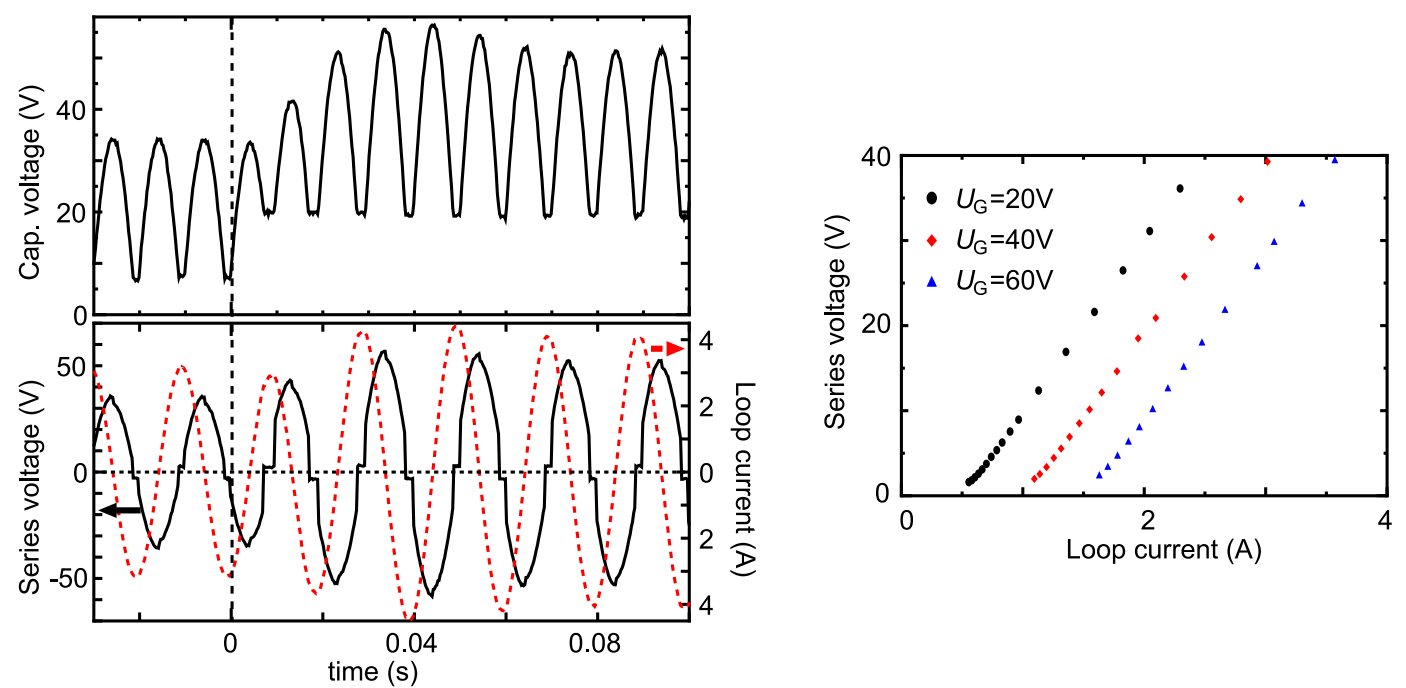

Figure 10: Experimental results when using MERS as a power flow controller. The left part shows the response when changing the minimum capacitor and by-pass length set-points. The right part shows the resulting loop current when changing the size of the injected series voltage.

systems since the first series capacitor installation at Ballston in 1928 [6]. The shaft failure at Mohave power station in 1970 gave awareness of the problem with sub-synchronous resonance [7]. Due to the limitation of series capacitors, new power electronics based technologies were developed and are referred to as Flexible AC Transmission System (FACTS) controllers [8].

MERS has been suggested as a new series FACTS controller [9]. The configuration shown in Fig. 9 is used to investigate the behavior of the MERS. In the case of load control, the MERS is operated to control the phase of the injected voltage of the phase of the current. When being used in power transmission, it is more important to control the size of the injected series voltage since this has a direct influence on the flow of power. Control of the size of the MERS voltage is performed by controlling the minimum capacitor voltage and the length of the by-pass period [9], by utilizing the active by-pass type of control (Fig. 2). In order to perform this control, it is necessary to have phase information of the current as well as direct measurement of the capacitor voltage. This leads to some more complexity compared to the load control applications.

Experimental results for the transmission system application are shown in Fig. 10. The left part shows the response with a step in set-point to the controller. It can be found that the new minimum capacitor voltage set-point is quickly reached. It takes approximately 2 cycles before the injected series voltage stabilizes. This is due to the MERS having similar characteristics to a series connection of a voltage source and a capacitor [9]. The right part of the figure shows the steady state loop current flow for various injected series voltages. Three different power source voltages were applied, leading to different resulting current for a given series voltage. By adjusting the size of the MERS series voltage, the flow of power can be controlled.

Important characteristics when using the MERS as a power flow controller are: 
- Large operating range, being able to inject up to rated series voltage also for low currents. This is the same capacitive voltage range as for the Static Synchronous Series Compensator, by using a simpler configuration.

- Line frequency switching is used, meaning high power semiconductors based on Gate Turn-off Thyristor technology can be used.

- Promising sub-synchronous characteristics, mainly acting as resistor for sub-harmonic frequencies [10].

- Potential for simple over-current protection by quickly entering parallel by-pass mode without destructive GTO turn-offs [11].

\section{Discussion}

Three different application areas of the MERS have been discussed. Firstly, series compensation has been shown to be a possible solution for load control concerning both voltage control and power factor correction. The suggested load control method controls the phase of the current into the lead area. This means reactive power is fed into the grid from the load. On one side, this is a benefit since most loads consume reactive power and local supply of reactive power can reduce transport of reactive power and resulting grid losses. On the other hand, if the load in an area is dominated by reactive power producing loads, care must be taken to make sure grid voltages are kept within their limits. Load control with series compensation means that the frequency can not be controlled and the application range becomes less than that of converter solutions. Applications such as fluorescent lamp intensity control seem promising, where MERS offers a simpler solution than that of the high frequency converter configurations.

Permanent magnet generators are getting more common with price reduction on permanent magnets as well as the coming of slow rotating generator applications such as in the area of wind power. Due to the inability to control the field excitation of these generators, the maximum output power is limited. MERS has been shown to be a possible solution to improve the output power. In principle a fixed series capacitor could be used to cancel half of the synchronous reactance of the generator and result in similar characteristics. Due to the resonance characteristics of the fixed series capacitor in series with an inductance, potential stability problems might occur; however, this has not been investigated in detail. If the generator system shall be able to operate during variable frequency, the use of fixed series capacitors is difficult due to the changing reactance of the capacitor. Additional, having a variable compensation device means that the power going out of the generator can be actively controlled.

Fixed series capacitor has been used for many years in transmission systems and this is likely to continue. MERS is suggested as a new series FACTS device and is in this respect one of many technologies. Work is currently in progress to compare the MERS technology to the existing technologies.

\section{Conclusion}

The Magnetic Energy Recovery Switch(MERS) has been shown to be a possible solution for low power to high power applications. The size of the injected series voltage can with simple control be controlled from zero to rated voltage within the current rating of the device. Load control application seems promising for cases where control of the frequency is not a necessity, such as for fluorescent lamps. By using MERS in series with permanent magnet generators can the output power capability of the machine be increased. At the high power end, can the power flow be controlled and increased by using MERS for series compensation in transmission systems. In summary, the paper suggests a wider use of series compensation in electrical systems.

\section{References}

[1] K. Shimada, T. Takaku, T. Matsukawa, and R. Shimada. Bi-directional current switch with snubber regeneration using p-mosfets. Proc. International Power Electronics Conference, (4):102-103, April 2000.

[2] Jan A. Wiik, Taku Takaku, Fransisco Danang Wijaya, Tadayuki Kitahara, and Ryuichi Shimada. Improvement of synchronous generator characteristics using bi-directional current phase control switch. EPE-PEMC, 2006.

[3] Jan A. Wiik, Takanori Isobe, Taku Takaku, Tadayuki Kitahara, and Ruyichi Shimada. Application of series connected current control switches for power factor correction. PCIM Europe, 2006.

[4] S. Y. R. Hui, W. Yan, H. Chung, P. W. Tam, and G. Ho. Energy efficiency comparison of dimmable electromagnetic and electronic ballast systems. IAS Annual Meeting, 2005. 
[5] T. Takaku, G. Homma, S. Igarashi, Y. Uchida, and R. Shimada. Power up and efficiency improvement of wind turbine generator by magnetic energy recovery switch. IPEC-Niigata 2005, 2005.

[6] E. K. Shelton. The series capacitor installation at ballston. General Electric Review, 31:432-434, 1928.

[7] M. C. Hall and D. A. Hodges. Experience with 500kv subsynchronous resonance and resulting turbine generator shaft damage at mohave generating station. IEEE Publication 76 CHIO66-O$P W R$, pages 22-29, 1976.

[8] N. G. Hingorani and L. Gyugyi. Understanding FACTS, Concepts and Technology of Flexible AC Transmission Systems. IEEE Press, 1999.

[9] Jan A. Wiik, F. Danang Widjaya, Takanori Isobe, Tadayuki Kitahara, and Ryuichi Shimada. Series connected power flow control using magnetic energy recovery switch (mers). PCC Nagoya, 2007.

[10] Jan A. Wiik, F. Danang Wijaya, and Ryuichi Shimada. An innovative series connected power flow controller, magnetic energy recovery switch (mers). PES General Meeting, 2007.

[11] Jan A. Wiik, F. Danang Wijaya, and Ryuichi Shimada. Robust series compensation in power systems using magnetic energy recovery switch (mers). IEEJ, SPC-07-70, June 2007. 